\title{
Supercloseness of finite element method on a Bakhvalov-type mesh for a singularly perturbed problem with two parameters *
}

\author{
Jin Zhang, ${ }^{\mathrm{a}, *}$, Yanhui Lv ${ }^{\mathrm{a}, 1}$ \\ ${ }^{a}$ School of Mathematics and Statistics, Shandong Normal University, Jinan 250014, China
}

\begin{abstract}
In this paper, the linear finite element method on a Bakhvalov-type mesh is applied to a singularly perturbed problem with two parameters. The solution of the problem exists two exponential boundary layers. A new interpolation, which is simple in construction and analysis, is introduced for convergence analysis. Furthermore, we find a subtle relationship between the Bakhvalov-type mesh itself and the weaker exponential layer and obtain an interesting result. Finally, we prove a supercloseness result between the Lagrange interpolation and the numerical solution. Numerical tests confirm our theoretical results.

Keywords: Singular perturbation, Convection-diffusion equation, Two

parameters, Finite element method, Bakhvalov-type mesh, Supercloseness
\end{abstract}

\section{Introduction}

We consider the two-parameter singularly perturbed boundary value problem

$$
-\varepsilon_{1} u^{\prime \prime}+\varepsilon_{2} b(x) u^{\prime}+c(x) u=f(x) \quad \text { in } \Omega:=(0,1), \quad u(0)=u(1)=0,
$$

${ }^{\star}$ This research is supported by National Natural Science Foundation of China (11771257, 11601251).

*Corresponding author: jinzhangalex@hotmail.com

${ }^{1}$ Email: yanhuilv@hotmail.com

Preprint submitted to Elsevier

January 5, 2021 
with

$$
\begin{aligned}
b(x) \geq \lambda>0, \quad c(x) \geq \beta>0 \quad & \text { on } \bar{\Omega}, \\
c(x)-\frac{1}{2} \varepsilon_{2} b^{\prime}(x) \geq \gamma>0 \quad & \text { on } \bar{\Omega},
\end{aligned}
$$

where $b, c, f$ are sufficiently smooth functions on $\bar{\Omega}=[0,1]$ and $\lambda, \beta, \gamma$ are constants. Here we are interested in the case $0<\varepsilon_{1}, \varepsilon_{2} \ll 1$ (see [8]). Thus the problem (1) is singularly perturbed. Moreover, the conditions (2) and (3) ensure that there exists a unique solution $u \in H_{0}^{1}(\Omega)$, which is characterized by boundary layers at $x=0$ and $x=1$.

It is found that the values of $\varepsilon_{1}$ and $\varepsilon_{2}$ strongly affect the properties of boundary layers. When $\varepsilon_{1} \ll \varepsilon_{2}^{2} \ll 1$, the boundary layer in the vicinity of $x=1$ is stronger than the boundary layer at $x=0$, as is similar to the reactionconvection-diffusion type problem. When $\varepsilon_{2}^{2} \ll \varepsilon_{1} \ll 1$, the widths of the layers around $x=0$ and $x=1$ are almost the same, as holds for the reaction-diffusion case. And when $\varepsilon_{2}=1$, the problem becomes a single parameter problem and exhibits boundary layer only at $x=0$.

For singular perturbation problems, it is well known that standard numerical methods do not work. Therefore, layer-adapted meshes represented by Shishkin-type meshes and Bakhvalov-type meshes (see 7] ) are explored to solve these problems. However, different from Shishkin-type meshes, Bakhvalov-type meshes brings great difficulties in convergence analysis (see [9, §2]), so there are few articles on this kind of meshes at present. But, the existence of Bakhvalovtype meshes is of great significance, because numerical results of Bakhvalov-type meshes are often significantly better than those of Shishkin-type meshes.

In recent years, the study of supercloseness has attracted more and more attention, since the supercloseness results play an important role in the posterior error analysis as well as for improved approximations of the solution(see [5, 4, 10, 11, 3] ). However, only the supercloseness study on Shishkin-type meshes exists at present for two-parameter singular perturbation problems (see [17, 14, 12]), while the research on Bakhvalov-type meshes seems to be in a blank state due to the lack of powerful technical tools. 
In this paper, we obtain the supercloseness results of the linear finite element method on a Bakhvalov-type mesh for the two-parameter singularly perturbed problem. First, we use a new interpolation, which has a simple structure and is easy for convergence analysis on Bakhvalov-type meshes. Second, we find a subtle relationship between the Bakhvalov-type mesh itself and the weaker exponential layer. Using this relationship, we get an interesting result. In addition, we would like to emphasize that in the analysis we found that the difficulties of supercloseness were mainly caused by the weak exponential layer and the regular part, rather than being controlled by the strong exponential layer. This may shed light to the subsequent analysis on higher-dimensional problems.

Our paper is organized as follows. In Section 2 we present properties of the exact solution as well as its derivatives, then introduce a Bakhvalov-type mesh and its properties, and define the finite element method of our problem. In Section 3 we define an interpolation $\Pi u$ and prove interpolation error bounds for the Lagrange interpolation. Section 4 contains error bounds of $u^{I}-u^{N}$ and the main result on the uniform error bound in the energy norm.

In this paper, the value of $C$ is independent of the perturbation parameters $\varepsilon_{1}, \varepsilon_{2}$ and the number $N$. For a set $D \subset \mathbb{R}$, we use the standard notation for Banach spaces $L^{p}(D)$, Sobolev spaces $W^{k, p}(D), H^{k}(D)=W^{k, 2}(D)$. For $p=2$, we have the norm $\|\cdot\|_{D}$ for $\|\cdot\|_{L^{2}(D)}$, and the seminorm $|\cdot|_{1, D}$ for $\|\cdot\|_{H^{1}(D)}$. When $D=\Omega$ we drop the subscript $D$ from the notation for simplicity.

\section{Regularity, Bakhvalov-type mesh and finite element method}

\subsection{Characteristic of Solution}

According to [1, §2], we describe the characteristics of the solution to problem (11), which are usually needed for error estimations in the case of singularly perturbed problems.

In order to describe the layers in the solution of (1), the characteristic equa- 
tion is introduced

$$
-\varepsilon_{1} g^{2}(x)+\varepsilon_{2} b(x) g(x)+c(x)=0 .
$$

It has two real solutions $g_{0}(x)<0$ and $g_{1}(x)>0$ which characterize the layers. Let

$$
\mu_{0}=-\max _{0 \leq x \leq 1} g_{0}(x), \quad \mu_{1}=\min _{0 \leq x \leq 1} g_{1}(x),
$$

where

$$
\mu_{0}=-g_{0}\left(x_{*}\right)=\frac{-\varepsilon_{2} b_{*}+\sqrt{\varepsilon_{2}^{2} b_{*}^{2}+4 \varepsilon_{1} c_{*}}}{2 \varepsilon_{1}},
$$

with $b_{*}=b\left(x_{*}\right)$ and $c_{*}=c\left(x_{*}\right)$ for some $x_{*} \in[0,1]$. The later analysis needs the following properties of $\mu_{0}$ and $\mu_{1}$ (see [13])

$$
\begin{aligned}
& \mu_{0} \leq \mu_{1}, \quad \max \left\{\mu_{0}^{-1}, \varepsilon_{1} \mu_{1}\right\} \leq C\left(\varepsilon_{2}+\varepsilon_{1}^{\frac{1}{2}}\right), \\
& \varepsilon_{2} \mu_{0} \leq \lambda^{-1}\|c\|_{L^{\infty}(\Omega)}, \quad \varepsilon_{2}\left(\varepsilon_{1} \mu_{1}\right)^{-\frac{1}{2}} \leq C \varepsilon_{2}^{\frac{1}{2}} .
\end{aligned}
$$

The values $\mu_{0}$ and $\mu_{1}$ determine the decay of the boundary layers. The following lemma from [7] provides more details on the behavior of the solution of (11) and its derivatives.

Lemma 1. Let $b, c, f \in C^{q}(\bar{\Omega})$ for some $q \geq 1$ and let $p, \kappa \in(0,1)$ be arbitrary. Assume that

$$
q\left\|b^{\prime}\right\|_{L^{\infty}(\Omega)} \varepsilon_{2} \leq \kappa(1-p) .
$$

Then

$$
\left|u^{(k)}(x)\right| \leq C\left(1+\mu_{0}^{k} e^{-p \mu_{0} x}+\mu_{1}^{k} e^{-p \mu_{1}(1-x)}\right) \quad x \in \Omega,
$$

for $0 \leq k \leq q$.

Then, from [6] the solution $u$ of (10) has the representation

$$
u=S+E_{0}+E_{1},
$$

with

$$
\left|S^{(k)}\right| \leq C, \quad\left|E_{0}^{(k)}\right| \leq C \mu_{0}^{k} e^{-p \mu_{0} x}, \quad\left|E_{1}^{(k)}\right| \leq C \mu_{1}^{k} e^{-p \mu_{1}(1-x)}
$$

for $x \in \Omega$ and $0 \leq k \leq q$. 


\subsection{Bakhvalov-type mesh and finite element method}

Let $N \in \mathbb{N}, N \geq 16$, be divisible by 4 , and

$$
\sigma_{j}=\frac{\tau}{p \mu_{j}} \ln \mu_{j} \leq \frac{1}{4} \quad j=0,1,
$$

where $\tau \geq 1$ is a user-chosen parameter and $p \in(0,1)$ is the parameter from Lemma 1. We set $\sigma_{0}$ and $1-\sigma_{1}$ as Bakhvalov-type mesh's transition points. Transition points are the points where the mesh changes from fine to coarse and viceversa. Set $\Omega_{0}=\left(0, \sigma_{0}\right), \Omega_{e}=\left(\sigma_{0}, 1-\sigma_{1}\right)$ and $\Omega_{1}=\left(1-\sigma_{1}, 1\right)$. Then define a mesh on $\bar{\Omega}$ such that it is equidistant on $\bar{\Omega}_{e}$ with $N / 2$ mesh subintervals, and gradually divided on $\bar{\Omega}_{0}$ and $\bar{\Omega}_{1}$ with $N / 4$ mesh subintervals. Similar to [1], the mesh points $x_{i}, i=0,1, \cdots, N$, are defined by

$$
x_{i}=\left\{\begin{array}{l}
\frac{\tau}{p \mu_{0}} \varphi_{0}\left(t_{i}\right) \quad i=0,1, \cdots, \frac{N}{4}, \\
\sigma_{0}+2\left(t_{i}-\frac{1}{4}\right)\left(1-\sigma_{0}-\sigma_{1}\right) \quad i=\frac{N}{4}, \frac{N}{4}+1, \cdots, \frac{3 N}{4}, \\
1-\frac{\tau}{p \mu_{1}} \varphi_{1}\left(t_{i}\right) \quad i=\frac{3 N}{4}, \frac{3 N}{4}+1, \cdots, N,
\end{array}\right.
$$

where $t_{i}=\frac{i}{N}, i=0,1, \cdots, N$ and

$$
\varphi_{0}(t)=-\ln \left(1-4\left(1-\mu_{0}^{-1}\right) t\right), \quad \varphi_{1}(t)=-\ln \left(1-4\left(1-\mu_{1}^{-1}\right)(1-t)\right) .
$$

The mesh generating functions $\varphi_{0}$ and $\varphi_{1}$ are piecewise continuously differentiable and satisfy the following conditions

$$
\begin{aligned}
& \varphi_{0}(0)=0, \quad \varphi_{0}\left(\frac{1}{4}\right)=\ln \mu_{0}, \\
& \varphi_{1}\left(\frac{3}{4}\right)=\ln \mu_{1}, \quad \varphi_{1}(1)=0 .
\end{aligned}
$$

The weak form of problem (11) is to find $u \in H_{0}^{1}(\Omega)$ such that

$$
a(u, v)=(f, v) \quad \forall v \in H_{0}^{1}(\Omega)
$$

where

$$
a(u, v):=\varepsilon_{1}\left(u^{\prime}, v^{\prime}\right)+\varepsilon_{2}\left(b u^{\prime}, v\right)+(c u, v) \quad u, v \in H_{0}^{1}(\Omega),
$$

and $(\cdot, \cdot)$ denotes the standard scalar product in $L^{2}(\Omega)$. 
Set $I_{i}:=\left[x_{i}, x_{i+1}\right]$ with $i=0,1, \cdots, N-1$. Define the linear finite element space on the Bakhvalov-type mesh (9)

$$
V^{N}=\left\{w \in C(\bar{\Omega}): w(0)=w(1)=0,\left.w\right|_{I_{i}} \in P_{1}\left(I_{i}\right) \text { for } i=0, \ldots, N-1\right\}
$$

with the standard basis $\left\{\theta_{i}\right\}_{i=0}^{N}$ of hat functions, i.e., $\theta_{i}\left(x_{j}\right)=\delta_{i j}, i, j=0,1, \cdots, N$. The finite element method for (10) reads as: Find $u^{N} \in V^{N}$ such that

$$
a\left(u^{N}, v^{N}\right)=\left(f, v^{N}\right) \quad \forall v^{N} \in V^{N}
$$

The energy norm associated with $a(\cdot, \cdot)$ is defined by

$$
\|v\|_{E}^{2}:=\varepsilon_{1}|v|_{1}^{2}+\|v\|^{2} \quad \forall v \in H^{1}(\Omega)
$$

Using (3), it is easy to see that one has the coercivity

$$
a\left(v^{N}, v^{N}\right) \geq \alpha\left\|v^{N}\right\|_{E}^{2} \quad \text { for all } v^{N} \in V^{N},
$$

with $\alpha=\min (1, \gamma)$. It follows that $u^{N}$ is well defined by (11) (see [2] and references therein). Hence, the problem (11) has a unique solution.

\subsection{Some preliminary results}

In this subsection, we present some preliminary results on the Bakhvalovtype mesh (9).

Since $1 \ll \mu_{0} \leq \mu_{1}$, throughout the paper we assume

$$
\mu_{1}^{-1} \leq \mu_{0}^{-1} \leq N^{-1}
$$

which is not a restriction in practice.

Remark 1. Without assumption (12), there will be $\mu_{0}^{-1}>N^{-1}$ or $\mu_{1}^{-1}>N^{-1}$. We might as well take $\mu_{0}^{-1}>N^{-1}$, then the boundary layer at $x=0$ can be captured by standard triangulation and it is unnecessary for us to use finer meshes in the layer area.

Similar to Lemma 2 in [15], one has the following two lemmas. 
Lemma 2. The mesh sizes $h_{i}:=x_{i+1}-x_{i}, i=0,1, \cdots, N-1$, satisfy

$$
\begin{aligned}
& h_{0} \leq h_{1} \leq \cdots \leq h_{\frac{N}{4}-2}, \\
& \frac{\tau}{4 p} \mu_{0}^{-1} \leq h_{\frac{N}{4}-2} \leq \frac{\tau}{p} \mu_{0}^{-1}, \\
& \frac{\tau}{2 p} \mu_{0}^{-1} \leq h_{\frac{N}{4}-1} \leq \frac{4 \tau}{p} N^{-1}, \\
& N^{-1} \leq h_{i} \leq 2 N^{-1} \quad \frac{N}{4} \leq i \leq \frac{3 N}{4}-1, \\
& \frac{\tau}{2 p} \mu_{1}^{-1} \leq h_{\frac{3 N}{4}} \leq \frac{4 \tau}{p} N^{-1}, \\
& \frac{\tau}{4 p} \mu_{1}^{-1} \leq h_{\frac{3 N}{4}+1} \leq \frac{\tau}{p} \mu_{1}^{-1}, \\
& h_{\frac{3 N}{4}+1} \geq h_{\frac{3 N}{4}+2} \geq \cdots \geq h_{N-1} .
\end{aligned}
$$

Lemma 3. On the Bakhvalov-type mesh (9), we obtain for $0 \leq m \leq \tau$

$$
\begin{aligned}
& h_{i}^{m} e^{-p \mu_{0} x_{i}} \leq C \mu_{0}^{-m} N^{-m} \quad i=0,1, \cdots, \frac{N}{4}-2, \\
& h_{i}^{m} e^{-p \mu_{1}\left(1-x_{i+1}\right)} \leq C \mu_{1}^{-m} N^{-m} \quad i=\frac{3 N}{4}+1, \frac{N}{4}+2, \cdots, N-1 .
\end{aligned}
$$

Here we introduce a very interesting lemma as follows, which plays a key role in the later analysis.

Lemma 4. Assume $\tau \geq \frac{5}{2}$. On the Bakhvalov-type mesh (9), one has

$$
\max _{i=0,1, \cdots, \frac{N}{4}-3}\left\{\left(h_{i+1}-h_{i}\right) e^{-p \mu_{0} x_{i+1}}\right\} \leq C \mu_{0}^{-1} N^{-2} .
$$

Proof. Set $T_{i}=\left(h_{i+1}-h_{i}\right) e^{-p \mu_{0} x_{i+1}}$ for $i=0,1, \cdots, \frac{N}{4}-3$. From (9) we obtain

$$
\begin{aligned}
T_{i} & =\left(h_{i+1}-h_{i}\right) e^{-p \mu_{0} x_{i+1}} \\
& =\frac{\tau}{p \mu_{0}} \ln \left(\frac{\left(1-\xi(i+1) N^{-1}\right)^{2}}{\left[1-\xi(i+2) N^{-1}\right]\left[1-\xi i N^{-1}\right]}\right) \cdot\left(1-\xi(i+1) N^{-1}\right)^{\tau} \\
& =C \mu_{0}^{-1} \ln \left(1+\frac{\xi^{2} N^{-2}}{\left[1-\xi(i+2) N^{-1}\right]\left[1-\xi i N^{-1}\right]}\right) \cdot\left(1-\xi(i+1) N^{-1}\right)^{\tau},
\end{aligned}
$$


where $\xi=4\left(1-\mu_{0}^{-1}\right)$. Thus we have

$$
\begin{aligned}
T_{0} & =C \mu_{0}^{-1} \ln \left(1+\frac{\xi^{2} N^{-2}}{\left(1-2 \xi N^{-1}\right)}\right) \cdot\left(1-\xi N^{-1}\right)^{\tau} \\
& \leq C \mu_{0}^{-1} \ln \left(1+\frac{\xi^{2} N^{-2}}{\left(1-2 \xi N^{-1}\right)}\right) \\
& \leq C \mu_{0}^{-1} \frac{\xi^{2} N^{-2}}{1-2 \xi N^{-1}} \\
& \leq C \mu_{0}^{-1} N^{-2}
\end{aligned}
$$

and from (12) we can get

$$
\begin{aligned}
& T_{\frac{N}{4}-3} \\
& =C \mu_{0}^{-1} \ln \left(1+\frac{\xi^{2} N^{-2}}{\left[1-\xi\left(\frac{1}{4}-N^{-1}\right)\right]\left[1-\xi\left(\frac{1}{4}-3 N^{-1}\right)\right]}\right) \cdot\left(1-\xi\left(\frac{1}{4}-2 N^{-1}\right)\right)^{\tau} \\
& =C \mu_{0}^{-1} \ln \left(1+\frac{\xi^{2} N^{-2}}{\left[\mu_{0}^{-1}+\xi N^{-1}\right)\left(\mu_{0}^{-1}+3 \xi N^{-1}\right]}\right) \cdot\left(\mu_{0}^{-1}+\xi N^{-1}\right)^{\tau} \\
& \leq C \mu_{0}^{-1} \ln \left(1+\frac{1}{3}\right) \cdot\left(N^{-1}+\xi N^{-1}\right)^{\tau} \\
& \leq C \mu_{0}^{-1} N^{-\tau} \leq C \mu_{0}^{-1} N^{-\frac{5}{2}} .
\end{aligned}
$$

Next, we set $T(s)=C \mu_{0}^{-1} \ln \left(1+\frac{\xi^{2} N^{-2}}{\left[1-\xi(s+2) N^{-1}\right]\left[1-\xi s N^{-1}\right]}\right) \cdot\left(1-\xi(s+1) N^{-1}\right)^{\tau}$ for $s \in\left[0, \frac{N}{4}-3\right]$, then we take the derivative of $T(s)$ to analyze the monotonicity of $T(s)$ :

$$
T^{\prime}(s)=M(2 Q(s)-\tau \ln (1+Q(s))),
$$

where $M=\xi N^{-1}\left(1-\xi(s+1) N^{-1}\right)^{\tau-1}$ and

$$
\begin{aligned}
Q(s) & =\frac{\xi^{2} N^{-2}}{\left(1-\xi(s+2) N^{-1}\right)\left(1-\xi s N^{-1}\right)} \\
& \in\left[\frac{\xi^{2} N^{-2}}{1-2 \xi N^{-1}}, \frac{\xi^{2} N^{-2}}{\left(\mu_{0}^{-1}+\xi N^{-1}\right)\left(\mu_{0}^{-1}+3 \xi N^{-1}\right)}\right] .
\end{aligned}
$$

Obviously $M>0$. So we just analyze the positive and negative properties of $2 Q(s)-\tau \ln (1+Q(s))$ and set

$$
F(t)=2 t-\tau \ln (1+t) \quad t \in[0,+\infty)
$$

After a brief analysis, we found that when $\tau \geq \frac{5}{2}, F(0)=0, F(t)<0$ in $\left[0, t_{*}\right)$ and $F(t) \geq 0$ in $\left[t_{*},+\infty\right]$ for some $t_{*} \in(0,+\infty)$. 
Therefore, when $\tau \geq \frac{5}{2}, T(s)$ decreases monotonously as $s$ increases, or decreases first and then increases, or increases monotonously. To sum up, we can get $\max _{s \in\left[0, \frac{N}{4}-3\right]}\{T(s)\}=\max \left\{T(0), T\left(\frac{N}{4}-3\right)\right\}$. And then combine (15) and (16) to get our conclusion.

\section{Interpolation errors}

Now we introduce a new interpolation defined in [15] for our uniform convergence and briefly describe the structure of this interpolation.

For any $v \in C^{0}(\bar{\Omega})$ its Lagrange interpolation $v^{I}$ on the Bakhvalov-type mesh (9) is defined by

$$
v^{I}=\sum_{i=0}^{N} v\left(x_{i}\right) \theta_{i}(x),
$$

where $\theta_{i}(x)$ is the piecewise linear polynomial satisfying the conditions $\theta_{i}\left(x_{j}\right)=$ $\delta_{i j}$ for $i, j=0, \ldots, N$. Here $\delta_{i j}$ is the Kronecker symbol. We define the interpolation $\Pi u$ to the solution $u$ by

$$
\Pi u=S^{I}+E_{0}^{I}+\pi E_{1},
$$

where $S^{I}$ and $E_{0}^{I}$ are the Lagrange interpolation to $S$ and $E_{0}$, respectively. And

$$
\pi E_{1}=\sum_{i=0}^{\frac{3 N}{4}} E_{1}\left(x_{i}\right) \theta_{i}(x)+\sum_{i=\frac{3 N}{4}+2}^{N} E_{1}\left(x_{i}\right) \theta_{i}(x) .
$$

Define

$$
\left(P E_{1}\right)(x)=E_{1}\left(x_{\frac{3 N}{4}+1}\right) \theta_{\frac{3 N}{4}+1}(x),
$$

and clearly we have $\pi E_{1}, \Pi u \in V^{N}$ and

$$
\begin{aligned}
& \left(\pi E_{1}\right)(x)=E_{1}^{I}-\left(P E_{1}\right)(x), \\
& \Pi u=u^{I}-\left(P E_{1}\right)(x), \\
& \left.\pi E_{1}\right|_{\left[x_{0}, x_{\frac{3 N}{4}}\right] \cup\left[x_{\frac{3 N}{4}+2}, x_{N}\right]}=\left.E_{1}^{I}\right|_{\left[x_{0}, x_{\frac{3 N}{4}}\right] \cup\left[x_{\frac{3 N}{4}+2}, x_{N}\right]},
\end{aligned}
$$

where $E_{1}^{I}$ and $u^{I}$ are Lagrange interpolation of $E_{1}$ and $u^{I}$, respectively.

Similar to [15, (38)], the following lemma can be proved. 
Lemma 5. Assume $\tau \geq \frac{5}{2}$. Then one has

$$
\left\|\left(P E_{1}\right)(x)\right\|_{E} \leq C N^{-\frac{5}{2}}\left(\varepsilon_{2}+\varepsilon_{1}^{\frac{1}{2}}\right)^{\frac{1}{2}}+C N^{-3} .
$$

Remark 2. It can be seen from the definition that the new interpolation introduced by us has the advantages of simple structure and easy analysis compared with quasi-interpolation(see [1]). Moreover, our interpolation can be easily extended to general problems, such as higher-order finite element methods and higher-dimensional singular perturbation problems.

Next we will give some results of Lagrange interpolation. Standard interpolation theories yield

$$
\left\|v-v^{I}\right\|_{W^{l, q}\left(I_{i}\right)} \leq C h_{i}^{2-l+\frac{1}{q}-\frac{1}{p}}|v|_{W^{2, p}\left(I_{i}\right)},
$$

for all $v \in W^{2, p}\left(I_{i}\right), i=0,1, \cdots, N-1$, where $l=0,1$ and $1 \leq p, q \leq \infty$.

Lemma 6. Assume $\tau \geq \frac{5}{2}$. On the Bakhvalov-type mesh (91), one has

$$
\begin{gathered}
\left\|E_{j}-E_{j}^{I}\right\| \leq C N^{-\frac{5}{2}}, \quad j=0,1, \\
\left\|S-S^{I}\right\|+\left\|u-u^{I}\right\| \leq C N^{-2} .
\end{gathered}
$$

Proof. For $0 \leq i \leq \frac{N}{4}-2$, we use (21), (7) and (13) with $m=\frac{5}{2}$ to obtain

$$
\begin{aligned}
\sum_{i=0}^{\frac{N}{4}-2}\left\|E_{0}-E_{0}^{I}\right\|_{I_{i}}^{2} & \leq C \sum_{i=0}^{\frac{N}{4}-2} h_{i}^{4}\left\|E_{0}^{\prime \prime}\right\|_{I_{i}}^{2} \leq C \sum_{i=0}^{\frac{N}{4}-2} h_{i}^{5}\left\|E_{0}^{\prime \prime}\right\|_{L^{\infty}\left(I_{i}\right)}^{2} \\
& \leq C \sum_{i=0}^{\frac{N}{4}-2} h_{i}^{5} \mu_{0}^{4} e^{-2 p \mu_{0} x_{i}}=C \sum_{i=0}^{\frac{N}{4}-2} \mu_{0}^{4}\left(h_{i}^{\frac{5}{2}} e^{-p \mu_{0} x_{i}}\right)^{2} \\
& \leq C \mu_{0}^{-1} N^{-4}
\end{aligned}
$$

For $\frac{N}{4}-1 \leq i \leq N-1$, we use the triangle inequality, Lemma 2 and (77) to get

$$
\begin{aligned}
\sum_{i=\frac{N}{4}-1}^{N-1}\left\|E_{0}-E_{0}^{I}\right\|_{I_{i}}^{2} & \leq C \sum_{i=\frac{N}{4}-1}^{N-1} h_{i}\left\|E_{0}-E_{0}^{I}\right\|_{L^{\infty}\left(I_{i}\right)}^{2} \leq C \sum_{i=\frac{N}{4}-1}^{N-1} h_{i}\left\|E_{0}\right\|_{L^{\infty}\left(I_{i}\right)}^{2} \\
& \leq C \sum_{i=\frac{N}{4}-1}^{N-1} h_{i} e^{-2 p \mu_{0} x_{\frac{N}{4}}-1} \leq C \sum_{i=\frac{N}{4}-1}^{N-1} N^{-1} N^{-2 \tau} \\
& \leq C N^{-5} .
\end{aligned}
$$


From (12), we have

$$
\left\|E_{0}-E_{0}^{I}\right\| \leq C\left(\mu_{0}^{-1} N^{-4}+N^{-5}\right)^{\frac{1}{2}} \leq C N^{-\frac{5}{2}} .
$$

By similar arguments we can get the interpolation error of $E_{1}$. Standard arguments yield $\left\|S-S^{I}\right\| \leq C N^{-2}$. Thus we are done.

Lemma 7. Assume $\tau \geq \frac{5}{2}$. On the Bakhvalov-type mesh (9), one has

$$
\begin{aligned}
& \left|E_{j}-E_{j}^{I}\right|_{1} \leq C \mu_{j}^{\frac{1}{2}} N^{-1} \quad j=0,1, \\
& \left|S-S^{I}\right|_{1} \leq C N^{-1}
\end{aligned}
$$

Proof. For $0 \leq i \leq \frac{N}{4}-2$, from (21), (77) and (13) with $m=\frac{3}{2}$, we have

$$
\begin{aligned}
\sum_{i=0}^{\frac{N}{4}-2}\left|E_{0}-E_{0}^{I}\right|_{1, I_{i}}^{2} & \leq C \sum_{i=0}^{\frac{N}{4}-2} h_{i}^{2}\left\|E_{0}^{\prime \prime}\right\|_{I_{i}}^{2} \leq C \sum_{i=0}^{\frac{N}{4}-2} h_{i}^{3}\left\|E_{0}^{\prime \prime}\right\|_{L^{\infty}\left(I_{i}\right)}^{2} \\
& \leq C \sum_{i=0}^{\frac{N}{4}-2} h_{i}^{3} \mu_{0}^{4} e^{-2 p \mu_{0} x_{i}}=C \sum_{i=0}^{\frac{N}{4}-2} \mu_{0}^{4}\left(h_{i}^{\frac{3}{2}} e^{-p \mu_{0} x_{i}}\right)^{2} \\
& \leq C \sum_{i=0}^{\frac{N}{4}-2} \mu_{0}^{4} \mu_{0}^{-3} N^{-3} \leq C \mu_{0} N^{-2}
\end{aligned}
$$

For $i=\frac{N}{4}-1$, from the triangle inequality, the inverse inequality, (7) and Lemma 2 one has

$$
\begin{aligned}
&\left|E_{0}-E_{0}^{I}\right|_{1, I_{\frac{N}{4}-1}}^{2} \leq C\left|E_{0}\right|_{1, I_{\frac{N}{4}-1}}^{2}+C\left|E_{0}^{I}\right|_{1, I_{\frac{N}{4}-1}}^{2} \\
& \leq C \int_{\frac{x_{\frac{N}{4}-1}}{4}}^{x_{\frac{N}{4}}}\left(E_{0}^{\prime}\right)^{2} d x+h_{\frac{N}{4}-1}^{-2}\left\|E_{0}^{I}\right\|_{I_{\frac{N}{4}-1}}^{2} \\
& \leq C \int_{x_{\frac{N}{4}-1}^{\frac{N}{4}}}^{x_{0}} \mu_{0}^{2} e^{-2 p \mu_{0} x} d x+C h_{\frac{N}{4}-1}^{-1}\left\|E_{0}\right\|_{L^{\infty}\left(I_{\frac{N}{4}-1}\right)}^{2} \\
& \leq C \mu_{0} e^{-2 p \mu_{0} x_{\frac{N}{4}-1}}+C \mu_{0} e^{-2 p \mu_{0} x_{\frac{N}{4}-1}} \\
& \leq C \mu_{0} N^{-2 \tau} \leq C \mu_{0} N^{-5} .
\end{aligned}
$$


For $\frac{N}{4} \leq i \leq N-1$, from (21), (77) and Lemma 2, one has

$$
\begin{aligned}
\sum_{i=\frac{N}{4}}^{N-1}\left|E_{0}-E_{0}^{I}\right|_{1, I_{i}}^{2} & \leq C \sum_{i=\frac{N}{4}}^{N-1} h_{i}^{2}\left\|E_{0}^{\prime \prime}\right\|_{I_{i}}^{2} \leq C \sum_{i=\frac{N}{4}}^{N-1} h_{i}^{3}\left\|E_{0}^{\prime \prime}\right\|_{L^{\infty}\left(I_{i}\right)}^{2} \\
& \leq C \sum_{i=\frac{N}{4}}^{N-1} h_{i}^{3} \mu_{0}^{4} e^{-2 p \mu_{0} x_{i}} \leq C \sum_{i=\frac{N}{4}}^{N-1} N^{-3} \mu_{0}^{4} e^{-2 p \mu_{0} x} \frac{N}{4} \\
& \leq C N^{-2} \mu_{0}^{4} \mu_{0}^{-2 \tau} \leq C \mu_{0}^{-1} N^{-2} .
\end{aligned}
$$

Finally, we conclude

$$
\left|E_{0}-E_{0}^{I}\right|_{1} \leq C\left(\mu_{0} N^{-2}+\mu_{0} N^{-5}+C \mu_{0}^{-1} N^{-2}\right)^{\frac{1}{2}} \leq C \mu_{0}^{\frac{1}{2}} N^{-1} .
$$

The estimate for $\left|E_{1}-E_{1}^{I}\right|_{1}$ can be similarly derived. Standard arguments yield the bound of $\left|S-S^{I}\right|_{1}$.

From (4), Lemmas 6 and 7, we obtain the following theorem easily.

Theorem 1. Assume $\tau \geq \frac{5}{2}$. Then one has

$$
\left\|u-u^{I}\right\|_{E} \leq C\left(\varepsilon_{2}+\varepsilon_{1}^{\frac{1}{2}}\right)^{\frac{1}{2}} N^{-1}+C N^{-2} .
$$

\section{Uniform convergence}

Introduce $\chi:=\Pi u-u^{N}$. From (2.2), the Galerkin orthogonality, (6), (19) and integration by parts for $\int_{0}^{1} b\left(\pi E_{1}-E_{1}\right)^{\prime} \chi \mathrm{d} x$, one has

$$
\begin{aligned}
& \alpha\|\chi\|_{E}^{2} \leq a(\chi, \chi)=a(\Pi u-u, \chi) \\
& =\varepsilon_{1} \int_{0}^{1}\left(u^{I}-u\right)^{\prime} \chi^{\prime} d x-\varepsilon_{1} \int_{0}^{1}\left(P E_{1}\right)^{\prime} \chi^{\prime} d x \\
& +\varepsilon_{2} \int_{0}^{1} b\left(S^{I}-S\right)^{\prime} \chi d x+\varepsilon_{2} \int_{0}^{1} b\left(E_{0}^{I}-E_{0}\right)^{\prime} \chi d x \\
& -\varepsilon_{2} \int_{0}^{1} b\left(\pi E_{1}-E_{1}\right) \chi^{\prime} d x-\varepsilon_{2} \int_{0}^{1} b^{\prime}\left(\pi E_{1}-E_{1}\right) \chi d x \\
& +\int_{0}^{1} c\left(u^{I}-u\right) \chi d x-\int_{0}^{1} c\left(P E_{1}\right) \chi d x \\
& =I+I I+I I I+I V+V+V I+V I I+V I I I .
\end{aligned}
$$


In the following we will analyze each term in the right-hand side of (22).

From integration by parts we first prove

$$
I=0 .
$$

From Hölder inequalities, (18), Lemmas 5 and 6] we obtain

$$
\begin{aligned}
& |(I I+V I I I)+(V I+V I I)| \\
\leq & C\left\|P E_{1}\right\|_{E}\|\chi\|_{E}+C\left(\varepsilon_{2}\left\|E_{1}-E_{1}^{I}\right\|+\varepsilon_{2}\left\|P E_{1}\right\|+\left\|u-u^{I}\right\|\right)\|\chi\| \\
\leq & C N^{-2}\|\chi\|_{E} .
\end{aligned}
$$

Imitating the analysis in Lemma 4 in [16]. One has

$$
|I I I| \leq C \varepsilon_{2}^{\frac{1}{2}} N^{-2} \ln ^{\frac{1}{2}} N\|\chi\|_{E} .
$$

In the following two lemmas we will give the estimates of IV and V.

Lemma 8. Assume $\tau \geq \frac{5}{2}$. On the Bakhvalov-type mesh (91), one has

$$
|I V| \leq C\left(\varepsilon_{2}^{\frac{1}{2}} N^{-2} \ln ^{\frac{1}{2}} N+N^{-3}\right)\|\chi\|_{E} .
$$

Proof. For the sake of analysis, the term $\int_{0}^{1} b\left(E_{0}^{I}-E_{0}\right)^{\prime} \chi d x$ is separated into three parts as follows:

$$
\begin{aligned}
\int_{0}^{1} b\left(E_{0}^{I}-E_{0}\right)^{\prime} \chi d x & =\int_{x_{0}}^{x_{\frac{N}{4}}-1} b\left(E_{0}^{I}-E_{0}\right)^{\prime} \chi d x+\int_{x_{\frac{N}{4}-1}}^{x_{N}} b\left(E_{0}^{I}-E_{0}\right)^{\prime} \chi d x \\
& +\int_{x_{\frac{N}{4}}}^{x_{N}} b\left(E_{0}^{I}-E_{0}\right)^{\prime} \chi d x \\
& =: \Delta_{1}+\Delta_{2}+\Delta_{3} .
\end{aligned}
$$

For $\Delta_{1}$, we first use the integral identity (see [7, (5.14)]) to get

$$
\begin{aligned}
\int_{I_{i}}\left(E_{0}-E_{0}^{I}\right)^{\prime} \chi d x & =\frac{1}{6} \int_{I_{i}} E_{0}^{\prime \prime \prime}\left(J_{i}^{2}\right)^{\prime} \chi^{\prime} d x-\frac{1}{3}\left(\frac{h_{i}}{2}\right)^{2} \int_{I_{i}} E_{0}^{\prime \prime \prime} \chi d x \\
& +\left.\frac{1}{3}\left(\frac{h_{i}}{2}\right)^{2} E_{0}^{\prime \prime} \chi\right|_{x_{i}} ^{x_{i}+1}
\end{aligned}
$$


where $J_{i}(x)=\frac{1}{2}\left(x-x_{i}\right)\left(x-x_{i+1}\right)$. Then from (26) and Hölder inequalities we can obtain

$$
\begin{aligned}
\left|\Delta_{1}\right| & \leq C \sum_{i=0}^{\frac{N}{4}-2} h_{i}^{3}\left\|E_{0}^{\prime \prime \prime}\right\|_{I_{i}}\left\|\chi^{\prime}\right\|_{I_{i}}+C \sum_{i=0}^{\frac{N}{4}-2} h_{i}^{2}\left\|E_{0}^{\prime \prime \prime}\right\|_{I_{i}}\|\chi\|_{I_{i}} \\
& +C h_{0}^{2}\left|E_{0}^{\prime \prime}(0)\left\|\chi(0)\left|+C \sum_{i=0}^{\frac{N}{4}-3}\left(h_{i+1}-h_{i}\right)\left(h_{i+1}+h_{i}\right)\right| E_{0}^{\prime \prime}\left(x_{i+1}\right)\right\| \chi\left(x_{i+1}\right)\right| \\
& +C h_{\frac{N}{4}-2}^{2}\left|E_{0}^{\prime \prime}\left(x_{\frac{N}{4}-1}\right) \| \chi\left(x_{\frac{N}{4}-1}\right)\right| \\
& =: A_{1}+A_{2}+A_{3}+A_{4}+A_{5} .
\end{aligned}
$$

Using the inverse inequality, (77) and (13) with $m=\frac{5}{2}$ to obtain

$$
\begin{aligned}
A_{1} & \leq C \sum_{i=0}^{\frac{N}{4}-2} h_{i}^{3} h_{i}^{-\frac{1}{2}}\left\|E_{0}^{\prime \prime \prime}\right\|_{L^{\infty}\left(I_{i}\right)}\|\chi\|_{I_{i}} \leq C \sum_{i=0}^{\frac{N}{4}-2} h_{i}^{\frac{5}{2}} \mu_{0}^{3} e^{-p \mu_{0} x_{i}}\|\chi\|_{I_{i}} \\
& \leq C \sum_{i=0}^{\frac{N}{4}-2} \mu_{0}^{\frac{1}{2}} N^{-\frac{5}{2}}\|\chi\|_{I_{i}} \leq C \mu_{0}^{\frac{1}{2}}\left(\sum_{i=0}^{\frac{N}{4}-2} N^{-5}\right)^{\frac{1}{2}}\left(\sum_{i=0}^{\frac{N}{4}-2}\|\chi\|_{I_{i}}^{2}\right)^{\frac{1}{2}} \\
& \leq C \mu_{0}^{\frac{1}{2}} N^{-2}\|\chi\|_{\left[x_{0}, x_{\frac{N}{4}-1}\right]} .
\end{aligned}
$$

And to use (7) and (13) with $m=\frac{5}{2}$ again to get

$$
\begin{aligned}
A_{2} & \leq C \sum_{i=0}^{\frac{N}{4}-2} h_{i}^{2} h_{i}^{\frac{1}{2}}\left\|E_{0}^{\prime \prime \prime}\right\|_{L^{\infty}\left(I_{i}\right)}\|\chi\|_{I_{i}} \leq C \sum_{i=0}^{\frac{N}{4}-2} h_{i}^{\frac{5}{2}} \mu_{0}^{3} e^{-p \mu_{0} x_{i}}\|\chi\|_{I_{i}} \\
& \leq C \sum_{i=0}^{\frac{N}{4}-2} \mu_{0}^{\frac{1}{2}} N^{-\frac{5}{2}}\|\chi\|_{I_{i}} \leq C \mu_{0}^{\frac{1}{2}}\left(\sum_{i=0}^{\frac{N}{4}-2} N^{-5}\right)^{\frac{1}{2}}\left(\sum_{i=0}^{\frac{N}{4}-2}\|\chi\|_{I_{i}}^{2}\right)^{\frac{1}{2}} \\
& \leq C \mu_{0}^{\frac{1}{2}} N^{-2}\|\chi\|_{\left[x_{0}, x_{\frac{N}{4}-1}\right]} .
\end{aligned}
$$

Obviously we have $\chi \in V^{N}$, so one has

$$
A_{3}=0 \text {. }
$$


From (7), Lemmas 2, 4 and the inverse inequality we have

$$
\begin{aligned}
A_{4} & \leq C \sum_{i=0}^{\frac{N}{4}-3}\left(h_{i+1}-h_{i}\right)\left(h_{i+1}+h_{i}\right) \mu_{0}^{2} e^{-p \mu_{0} x_{i+1}}\left|\chi\left(x_{i+1}\right)\right| \\
& \leq C \mu_{0} N^{-2} \sum_{i=0}^{\frac{N}{4}-3}\left(h_{i+1}+h_{i}\right)\left|\chi\left(x_{i+1}\right)\right| \leq C \mu_{0} N^{-2} \sum_{i=0}^{\frac{N}{4}-3} h_{i+1}\|\chi\|_{L^{\infty}\left(I_{i+1}\right)} \\
& \leq C \mu_{0} N^{-2} \sum_{i=0}^{\frac{N}{4}-3} h_{i+1} h_{i+1}^{-\frac{1}{2}}\|\chi\|_{I_{i+1}} \\
& \leq C \mu_{0} N^{-2}\left(\sum_{i=0}^{\frac{N}{4}-3} h_{i+1}\right)^{\frac{1}{2}}\left(\sum_{i=0}^{\frac{N}{4}-3}\|\chi\|_{I_{i+1}}^{2}\right)^{\frac{1}{2}} \\
& \leq C \mu_{0} N^{-2} \mu_{0}^{-\frac{1}{2}} \ln ^{\frac{1}{2}} N\|\chi\|_{\left[x_{0}, x_{\frac{N}{4}-1}\right]} \\
& \leq C \mu_{0}^{\frac{1}{2}} N^{-2} \ln ^{\frac{1}{2}} N\|\chi\|_{\left[x_{0}, x_{\frac{N}{4}-1}\right]} .
\end{aligned}
$$

From (7), Lemma 2 and the inverse inequality we can obtain

$$
\begin{aligned}
A_{5} & \leq C h_{\frac{N}{4}-2}^{2} \mu_{0}^{2} e^{-p \mu_{0} x_{\frac{N}{4}-1}}\left|\chi\left(x_{\frac{N}{4}-1}\right)\right| \leq C N^{-\tau}\|\chi\|_{L^{\infty}\left(I_{\frac{N}{4}-2}\right)} \\
& \leq C N^{-\frac{5}{2}} h_{\frac{N}{4}-2}^{-\frac{1}{2}}\|\chi\|_{\frac{N}{4}-2} \\
& \leq C \mu_{0}^{\frac{1}{2}} N^{-\frac{5}{2}}\|\chi\|_{I_{\frac{N}{4}-2}} .
\end{aligned}
$$

Thus from (27)- (31) we have

$$
\left|\Delta_{1}\right| \leq C \mu_{0}^{\frac{1}{2}} N^{-2} \ln ^{\frac{1}{2}} N\|\chi\|_{E,\left[x_{0}, x_{\frac{N}{4}-1}\right]} .
$$

For $\Delta_{2}$, using Hölder inequalities, the triangle inequality, the inverse inequality, Lemma 2 and (7) yield

$$
\begin{aligned}
\Delta_{2} & \leq C\left\|\left(E_{0}^{I}\right)^{\prime}\right\|_{I_{\frac{N}{4}-1}}\|\chi\|_{I_{\frac{N}{4}-1}}+C\left\|E_{0}^{\prime}\right\|_{I_{\frac{N}{4}-1}}\|\chi\|_{I_{\frac{N}{4}-1}} \\
& \leq C h_{\frac{N}{4}-1}^{-\frac{1}{2}}\left\|E_{0}^{I}\right\|_{L^{\infty}\left(I_{\frac{N}{4}-1}\right)}\|\chi\|_{I_{\frac{N}{4}-1}}+C h_{\frac{N}{4}-1}^{\frac{1}{2}}\left\|E_{0}^{\prime}\right\|_{L^{\infty}\left(I_{\frac{N}{4}-1}\right)}\|\chi\|_{I_{\frac{N}{4}-1}} \\
& \leq C \mu_{0}^{\frac{1}{2}} e^{-p \mu_{0} x_{\frac{N}{4}-1}}\|\chi\|_{I_{\frac{N}{4}-1}}+C N^{-\frac{1}{2}} \mu_{0} e^{-p \mu_{0} x_{\frac{N}{4}-1}}\|\chi\|_{I_{\frac{N}{4}-1}} \\
& \leq C \mu_{0}^{\frac{1}{2}} N^{-\tau}\|\chi\|_{\frac{N}{4}-1}+C \mu_{0} N^{-\left(\frac{1}{2}+\tau\right)}\|\chi\|_{I_{\frac{N}{4}-1}} \\
& \leq C \mu_{0}^{\frac{1}{2}} N^{-\frac{5}{2}}\|\chi\|_{\frac{N}{4}-1}+C \mu_{0} N^{-3}\|\chi\|_{I_{\frac{N}{4}-1}} .
\end{aligned}
$$


For $\Delta_{3}$, using Hölder inequalities, (21), (7) and Lemma 2 to obtain

$$
\begin{aligned}
\Delta_{3} & \leq C \sum_{i=\frac{N}{4}}^{N-1}\left\|\left(E_{0}^{I}-E_{0}\right)^{\prime}\right\|_{I_{i}}\|\chi\|_{I_{i}} \leq C \sum_{i=\frac{N}{4}}^{N-1} h_{i}^{\frac{3}{2}}\left\|E_{0}^{\prime \prime}\right\|_{L^{\infty}\left(I_{i}\right)}\|\chi\|_{I_{i}} \\
& \leq C \sum_{i=\frac{N}{4}}^{N-1} h_{i}^{\frac{3}{2}} \mu_{0}^{2} e^{-p \mu_{0} x_{\frac{N}{4}}}\|\chi\|_{I_{i}} \leq C \sum_{i=\frac{N}{4}}^{N-1} h_{i}^{\frac{3}{2}} \mu_{0}^{2} \mu_{0}^{-\tau}\|\chi\|_{I_{i}} \\
& \leq C \sum_{i=\frac{N}{4}}^{N-1} N^{-\frac{3}{2}} \mu_{0}^{-\frac{1}{2}}\|\chi\|_{I_{i}} \leq C \mu_{0}^{-\frac{1}{2}}\left(\sum_{i=\frac{N}{4}}^{N-1} N^{-3}\right)^{\frac{1}{2}}\left(\sum_{i=\frac{N}{4}}^{N-1}\|\chi\|_{I_{i}}^{2}\right)^{\frac{1}{2}} \\
& \leq C \mu_{0}^{-\frac{1}{2}} N^{-1}\|\chi\|_{\left[x_{\frac{N}{4}}, x_{N}\right]}
\end{aligned}
$$

Therefore from (32)-(34) and (12) we have

$$
\left|\int_{0}^{1} b\left(E_{0}^{I}-E_{0}\right)^{\prime} \chi d x\right| \leq C\left(\mu_{0}^{\frac{1}{2}} N^{-2} \ln ^{\frac{1}{2}} N+\mu_{0} N^{-3}\right)\|\chi\| .
$$

Combine that with (5) we get

$$
\left|\varepsilon_{2} \int_{0}^{1} b\left(E_{0}^{I}-E_{0}\right)^{\prime} \chi d x\right| \leq C\left(\varepsilon_{2}^{\frac{1}{2}} N^{-2} \ln ^{\frac{1}{2}} N+N^{-3}\right)\|\chi\|_{E} .
$$

Lemma 9. Assume $\tau \geq \frac{5}{2}$ and let $\pi E_{1}$ be defined in (17). On the Bakhvalovtype mesh (9), one has

$$
|V I I| \leq C \varepsilon_{2}^{\frac{1}{2}} N^{-2}\|\chi\|_{E} .
$$

Proof. According to (20), the term $\int_{0}^{1} b\left(\pi E_{1}-E_{1}\right) \chi^{\prime} d x$ is separated into three parts as follows:

$$
\begin{aligned}
& \int_{0}^{1} b\left(\pi E_{1}-E_{1}\right) \chi^{\prime} d x \\
& =\int_{x_{0}}^{x^{\frac{3 N}{4}}} b\left(E_{1}^{I}-E_{1}\right) \chi^{\prime} d x+\int_{x_{\frac{3 N}{4}}}^{x^{\frac{3 N}{4}+2}} b\left(\pi E_{1}-E_{1}\right) \chi^{\prime} d x+\int_{x_{\frac{3 N}{4}+2}^{x_{N}}}^{x^{2}} b\left(E_{1}^{I}-E_{1}\right) \chi^{\prime} d x \\
& =\Gamma_{1}+\Gamma_{2}+\Gamma_{3} .
\end{aligned}
$$


For $\Gamma_{1}$, from Hölder inequalities, (21), (77) and Lemma 2, we have

$$
\begin{aligned}
\left|\Gamma_{1}\right| & \leq C \sum_{i=0}^{\frac{3 N}{4}-1}\left\|E_{0}^{I}-E_{0}\right\|_{I_{i}}\left\|\chi^{\prime}\right\|_{I_{i}} \leq C \sum_{i=0}^{\frac{3 N}{4}-1} h_{i}^{\frac{5}{2}}\left\|E_{1}^{\prime \prime}\right\|_{L^{\infty}\left(I_{i}\right)}\left\|\chi^{\prime}\right\|_{I_{i}} \\
& \leq C \sum_{i=0}^{\frac{3 N}{4}-1} h_{i}^{\frac{5}{2}} \mu_{1}^{2} e^{-p \mu_{1}\left(1-x_{\frac{N}{4}}\right)}\left\|\chi^{\prime}\right\|_{I_{i}} \leq C \sum_{i=0}^{\frac{3 N}{4}-1} N^{-\frac{5}{2}} \mu_{1}^{2} \mu_{1}^{-\tau}\left\|\chi^{\prime}\right\|_{I_{i}} \\
& \leq C \sum_{i=0}^{\frac{3 N}{4}-1} N^{-\frac{5}{2}} \mu_{1}^{-\frac{1}{2}}\left\|\chi^{\prime}\right\|_{I_{i}} \leq C \mu_{1}^{-\frac{1}{2}}\left(\sum_{i=0}^{\frac{3 N}{4}-1} N^{-5}\right)^{\frac{1}{2}}\left(\sum_{i=0}^{\frac{3 N}{4}-1}\left\|\chi^{\prime}\right\|_{I_{i}}^{2}\right)^{\frac{1}{2}} \\
& \leq C \mu_{1}^{-\frac{1}{2}} N^{-2}\left\|\chi^{\prime}\right\|_{\left[x_{0}, x_{\frac{3 N}{4}}\right]} \\
& \leq C \varepsilon_{1}^{-\frac{1}{2}} \mu_{1}^{-\frac{1}{2}} N^{-2}\|\chi\|_{E,\left[x_{0}, x_{\frac{3 N}{4}}\right]} .
\end{aligned}
$$

Now we analyze the term $\Gamma_{2}$. Note $\pi E_{1}=E_{1}^{I}-\left(P E_{1}\right)(x)$ on $\left[x_{\frac{3 N}{4}+1}, x_{\frac{3 N}{4}+2}\right]$ and from Hölder inequalities, (21), (17), (14) with $m=\frac{5}{2}$ and Lemma 2, one has

$$
\begin{aligned}
& \left|\int_{x_{\frac{3 N}{4}+1}}^{x_{\frac{3 N}{4}+2}} b\left(\pi E_{1}-E_{1}\right) \chi^{\prime} d x\right| \leq C \int_{x_{\frac{3 N}{4}+1}}^{x_{\frac{3 N}{4}+2}}\left|E_{1}^{I}-E_{1} \| \chi^{\prime}\right| d x \\
& +C\left|E_{1}\left(x_{\frac{3 N}{4}+1}\right)\right| \int_{x_{\frac{3 N}{4}+1}}^{x_{\frac{3 N}{4}+2}}\left|\theta_{\frac{3 N}{4}+1}(x) \chi^{\prime}\right| d x \\
& \leq C\left(\left\|E_{1}^{I}-E_{1}\right\|_{\frac{3 N}{4}+1}+\left|E_{1}\left(x_{\frac{3 N}{4}+1}\right)\right| h_{\frac{3 N}{4}+1}^{\frac{1}{2}}\right)\left\|\chi^{\prime}\right\|_{I_{\frac{3 N}{4}+1}} \\
& \leq C\left(h_{\frac{3 N}{4}+1}^{\frac{5}{2}}\left\|E_{1}^{\prime \prime}\right\|_{L^{\infty}\left(I_{\frac{3 N}{4}+1}\right)}+N^{-\tau} \mu_{1}^{-\frac{1}{2}}\right)\left\|\chi^{\prime}\right\|_{\frac{3 N}{4}+1} \\
& \leq C\left(h_{\frac{3 N}{4}+1}^{\frac{5}{2}} \mu_{1}^{2} e^{-p \mu_{1}\left(1-x_{\frac{3 N}{4}+2}\right)}+N^{-\tau} \mu_{1}^{-\frac{1}{2}}\right)\left\|\chi^{\prime}\right\|_{\frac{3 N}{4}+1} \\
& \leq C\left(\mu_{1}^{-\frac{1}{2}} N^{-\frac{5}{2}}+N^{-\frac{5}{2}} \mu_{1}^{-\frac{1}{2}}\right)\left\|\chi^{\prime}\right\|_{\frac{3 N}{4}+1} \\
& \leq C \varepsilon_{1}^{-\frac{1}{2}} \mu_{1}^{-\frac{1}{2}} N^{-\frac{5}{2}}\|\chi\|_{E, I_{\frac{3 N}{4}+1}} .
\end{aligned}
$$

On $\left[x_{\frac{3 N}{4}}, x_{\frac{3 N}{4}+1}\right]$, we have $\pi E_{1}=E_{1}\left(x_{\frac{3 N}{4}}\right) \theta_{\frac{3 N}{4}}(x)$ from (17). Thus from Hölder 
inequalities, (77) and Lemma 2] we have

$$
\begin{aligned}
& \left|\int_{x_{\frac{3 N}{4}}}^{x} x_{\frac{3 N}{4}+1} b\left(\pi E_{1}-E_{1}\right) \chi^{\prime} d x\right| \\
& \leq C\left|E_{1}\left(x_{\frac{3 N}{4}}\right)\right| \int_{x_{\frac{3 N}{4}}}^{x_{\frac{3 N}{4}}+1}\left|\theta_{\frac{3 N}{4}}(x)\left\|\chi^{\prime}\left|d x+C \int_{x_{\frac{3 N}{4}}}^{x_{\frac{3 N}{4}+1}}\right| E_{1}\right\| \chi^{\prime}\right| d x \\
& \leq C\left(\mid E_{1}\left(x_{\frac{3 N}{4}}\right)\left\|\theta_{\frac{3 N}{4}}(x)\right\|_{\frac{3 N}{4}}+\left\|E_{1}\right\|_{\frac{3 N}{4}}\right)\left\|\chi^{\prime}\right\|_{\frac{3 N}{4}} \\
& \leq C\left(\mu_{1}^{-\tau} h_{\frac{3 N}{4}}^{\frac{1}{2}}+\mu_{1}^{-\frac{1}{2}} N^{-\tau}\right)\left\|\chi^{\prime}\right\|_{\frac{3 N}{4}} \\
& \leq C\left(\mu_{1}^{-\frac{5}{2}} N^{-\frac{1}{2}}+\mu_{1}^{-\frac{1}{2}} N^{-\frac{5}{2}}\right)\left\|\chi^{\prime}\right\|_{\frac{I_{N}}{4}} \\
& \leq C \varepsilon_{1}^{-\frac{1}{2}}\left(\mu_{1}^{-\frac{5}{2}} N^{-\frac{1}{2}}+\mu_{1}^{-\frac{1}{2}} N^{-\frac{5}{2}}\right)\|\chi\|_{E, I_{\frac{3 N}{4}}} .
\end{aligned}
$$

Therefore, from (36) and (37) we can obtain

$$
\left|\Gamma_{2}\right| \leq C \varepsilon_{1}^{-\frac{1}{2}}\left(\mu_{1}^{-\frac{5}{2}} N^{-\frac{1}{2}}+\mu_{1}^{-\frac{1}{2}} N^{-\frac{5}{2}}\right)\|\chi\|_{E,\left[x_{\frac{3 N}{4}}, x_{\frac{3 N}{4}+2}\right]} .
$$

For $\Gamma_{3}$, from Hölder inequalities, (21), (14) with $m=\frac{5}{2}$, we have

$$
\begin{aligned}
\left|\Gamma_{3}\right| & \leq C \sum_{i=\frac{3 N}{4}+2}^{N-1}\left\|E_{1}^{I}-E_{1}\right\|_{I_{i}}\left\|\chi^{\prime}\right\|_{I_{i}} \leq C \sum_{i=\frac{3 N}{4}+2}^{N-1} h_{i}^{\frac{5}{2}}\left\|E_{1}^{\prime \prime}\right\|_{L^{\infty}\left(I_{i}\right)}\left\|\chi^{\prime}\right\|_{I_{i}} \\
& \leq C \sum_{i=\frac{3 N}{4}+2}^{N-1} h_{i}^{\frac{5}{2}} \mu_{1}^{2} e^{-p \mu_{1}\left(1-x_{i+1}\right)}\left\|\chi^{\prime}\right\|_{I_{i}} \leq C \sum_{i=\frac{3 N}{4}+2}^{N-1} \mu_{1}^{-\frac{1}{2}} N^{-\frac{5}{2}}\left\|\chi^{\prime}\right\|_{I_{i}} \\
& \leq C \mu_{1}^{-\frac{1}{2}}\left(\sum_{i=\frac{3 N}{4}+2}^{N-1} N^{-5}\right)^{\frac{1}{2}}\left(\sum_{i=\frac{3 N}{4}+2}^{N-1}\left\|\chi^{\prime}\right\|_{I_{i}}^{2}\right)^{\frac{1}{2}} \\
& \leq C \mu_{1}^{-\frac{1}{2}} N^{-2}\left\|\chi^{\prime}\right\|_{\left[x_{\frac{3 N}{4}+2}, x_{N}\right]} \\
& \leq C \varepsilon_{1}^{-\frac{1}{2}} \mu_{1}^{-\frac{1}{2}} N^{-2}\|\chi\|_{E,\left[x_{\frac{3 N}{4}+2}, x_{N}\right]} .
\end{aligned}
$$

From (35), (38), (39), (12) and (5) we are done.

Now we are in a position to present our main results.

Theorem 2. Assume $\tau \geq \frac{5}{2}$. Let the mesh $\left\{x_{i}\right\}$ be the Bakhvalov-type mesh (91). Then we have

$$
\left\|u^{I}-u^{N}\right\|_{E} \leq C \varepsilon_{2}^{\frac{1}{2}} N^{-2} \ln ^{\frac{1}{2}} N+C N^{-2} .
$$


Proof. From (23), (24), (25) and Lemmas 80, we can easily obtain

$$
\|\chi\|_{E} \leq C \varepsilon_{2}^{\frac{1}{2}} N^{-2} \ln ^{\frac{1}{2}} N+C N^{-2} .
$$

Recalling that $\left\|u^{I}-u^{N}\right\|_{E} \leq C\|\chi\|_{E}+C\left\|P E_{1}\right\|_{E}$, then our conclusion can be drawn from (40) and Lemma 5 ,

Same to Theorem 4 in [1], we prove the optimal order of uniform convergence in the following theorem.

Theorem 3. Assume $\tau \geq \frac{5}{2}$. Let the mesh $\left\{x_{i}\right\}$ be the Bakhvalov-type mesh (9). Then we have

$$
\left\|u-u^{N}\right\|_{E} \leq C\left(\varepsilon_{2}+\varepsilon_{1}^{\frac{1}{2}}\right)^{\frac{1}{2}} N^{-1}+C N^{-2} .
$$

Proof. From Theorem 1 and Theorem 2 we can easily prove the result.

Remark 3. From Theorems 圆 and 3, we can see Theorem 圆 presents a supercloseness result when $\varepsilon_{2}+\varepsilon_{1}^{\frac{1}{2}}<C N^{-2}$.

\section{Numerical experimentation}

In this section, we present numerical examples to verify our theoretical findings. We focus on numerical experiments on supercloseness results. Here, a test problem in [1] is introduced, which is adequate for our purpose,

$$
-\varepsilon_{1} u^{\prime \prime}+\varepsilon_{2} u^{\prime}+u=\cos \pi x \quad x \in \Omega, \quad u(0)=u(1)=0,
$$

with the exact solution

$$
u(x)=a \cos \pi x+b \sin \pi x+A e^{-\mu_{0} x}+B e^{-\mu_{1}(1-x)},
$$

where

$$
\begin{aligned}
& a=\frac{\varepsilon_{1} \pi^{2}+1}{\varepsilon_{2}^{2} \pi^{2}+\left(\varepsilon_{1} \pi^{2}+1\right)^{2}}, \quad b=\frac{\varepsilon_{2} \pi}{\varepsilon_{2}^{2} \pi^{2}+\left(\varepsilon_{1} \pi^{2}+1\right)^{2}}, \\
& A=-a \frac{1+e^{-\mu_{1}}}{1-e^{-\mu_{0}-\mu_{1}}}, \quad B=a \frac{1+e^{-\mu_{0}}}{1-e^{-\mu_{0}-\mu_{1}}}, \quad \mu_{0,1}=\frac{\mp \varepsilon_{2}+\sqrt{\varepsilon_{2}^{2}+4 \varepsilon_{1}}}{2 \varepsilon_{1}} .
\end{aligned}
$$


In order that (8) and (12) hold true, considering $16 \leq N \leq 2048$ in our numerical experiments and we take

$$
\begin{aligned}
& 0<\varepsilon_{1} \leq 10^{-7}, \quad 0<\varepsilon_{2} \leq 10^{-4}, \\
& p=0.5, \quad \tau=2 .
\end{aligned}
$$

We also consider $\varepsilon_{1}=1,10^{-2}, 10^{-4}, 10^{-6}$ or $\varepsilon_{2}=1$ to check the according numerical performances. For a fixed $\varepsilon_{1}, \varepsilon_{2}$ and $N$, we consider the errors

$$
e^{N}=\left\|u-u^{N}\right\|_{E}, \quad e_{I}^{N}=\left\|u^{I}-u^{N}\right\|_{E}
$$

and the corresponding rates of convergence

$$
p^{N}=\frac{\ln e^{N}-\ln e^{2 N}}{\ln 2}, \quad p_{I}^{N}=\frac{\ln e_{I}^{N}-\ln e_{I}^{2 N}}{\ln 2} .
$$

In Tables 10 we present the errors $e^{N}, e_{I}^{N}$ and the convergence orders $p^{N}$, $p_{I}^{N}$ for $\varepsilon_{1}=1,10^{-2}, 10^{-6}, 10^{-8}, 10^{-10}$ and $N=16, \ldots, 2048$ in the case of $\varepsilon_{2}=1,10^{-4}, 10^{-8}$.

The data show that no matter $\varepsilon_{2}$ is taken as a large value or a small value, there exists supercloseness results as long as $\varepsilon_{1}$ and $\varepsilon_{2}$ satisfy proper conditions. And we can see in some cases, some orders of $\left\|u-u^{N}\right\|_{E}$ are closer ones of $\left\|u^{I}-u^{N}\right\|_{E}$ such as the last two columns in Tables 36, so the supercloseness is very weak. These phenomena support Theorem 2

\section{References}

[1] M. Brdar and H. Zarin. A singularly perturbed problem with two parameters on a Bakhvalov-type mesh. J. Comput. Appl. Math., 292:307-319, 2016.

[2] S. C. Brenner and L. R. Scott. The mathematical theory of finite element methods, volume 15 of Texts in Applied Mathematics. Springer, New York, third edition, 2008. 
Table 1: $\left\|u-u^{N}\right\|_{E}$ in the case of $\varepsilon_{2}=1$

\begin{tabular}{c|ccccccccccccc}
\hline$N$ & $10^{0}$ & $10^{0}$ & $10^{-2}$ & $10^{-2}$ & $10^{-4}$ & $10^{-4}$ & $10^{-6}$ & $10^{-6}$ & $10^{-8}$ & $10^{-8}$ & $10^{-10}$ & $10^{-10}$ \\
\hline & & & & & & & & & & & & & \\
\hline & $e^{N}$ & $p^{N}$ & $e^{N}$ & $p^{N}$ & $e^{N}$ & $p^{N}$ & $e^{N}$ & $p^{N}$ & $e^{N}$ & $p^{N}$ \\
\hline 16 & $0.12 \mathrm{E}-1$ & 1.00 & $0.41 \mathrm{E}-1$ & 1.06 & $0.35 \mathrm{E}-1$ & 0.96 & $0.35 \mathrm{E}-1$ & 0.95 & $0.35 \mathrm{E}-1$ & 0.95 & $0.35 \mathrm{E}-1$ & 0.95 \\
32 & $0.62 \mathrm{E}-2$ & 1.00 & $0.20 \mathrm{E}-1$ & 1.01 & $0.18 \mathrm{E}-1$ & 1.00 & $0.18 \mathrm{E}-1$ & 1.00 & $0.18 \mathrm{E}-1$ & 1.00 & $0.18 \mathrm{E}-1$ & 1.00 \\
64 & $0.31 \mathrm{E}-2$ & 1.00 & $0.98 \mathrm{E}-2$ & 1.00 & $0.90 \mathrm{E}-2$ & 1.00 & $0.90 \mathrm{E}-2$ & 1.00 & $0.90 \mathrm{E}-2$ & 1.00 & $0.90 \mathrm{E}-2$ & 1.00 \\
128 & $0.16 \mathrm{E}-2$ & 1.00 & $0.49 \mathrm{E}-2$ & 1.00 & $0.45 \mathrm{E}-2$ & 1.00 & $0.45 \mathrm{E}-2$ & 1.00 & $0.45 \mathrm{E}-2$ & 1.00 & $0.45 \mathrm{E}-2$ & 1.00 \\
256 & $0.78 \mathrm{E}-3$ & 1.00 & $0.25 \mathrm{E}-2$ & 1.00 & $0.22 \mathrm{E}-2$ & 1.00 & $0.22 \mathrm{E}-2$ & 1.00 & $0.22 \mathrm{E}-2$ & 1.00 & $0.22 \mathrm{E}-2$ & 1.00 \\
512 & $0.39 \mathrm{E}-3$ & 1.00 & $0.12 \mathrm{E}-2$ & 1.00 & $0.11 \mathrm{E}-2$ & 1.00 & $0.11 \mathrm{E}-2$ & 1.00 & $0.11 \mathrm{E}-2$ & 1.00 & $0.11 \mathrm{E}-2$ & 1.00 \\
1024 & $0.19 \mathrm{E}-3$ & 1.00 & $0.62 \mathrm{E}-3$ & 1.00 & $0.56 \mathrm{E}-3$ & 1.00 & $0.56 \mathrm{E}-3$ & 1.00 & $0.56 \mathrm{E}-3$ & 1.00 & $0.56 \mathrm{E}-3$ & 1.00 \\
2048 & $0.97 \mathrm{E}-4$ & 1.00 & $0.31 \mathrm{E}-3$ & 1.00 & $0.28 \mathrm{E}-3$ & 1.00 & $0.28 \mathrm{E}-3$ & 1.00 & $0.28 \mathrm{E}-3$ & 1.00 & $0.28 \mathrm{E}-3$ & 1.00 \\
4096 & $0.49 \mathrm{E}-4$ & - & $0.15 \mathrm{E}-3$ & - & $0.14 \mathrm{E}-3$ & - & $0.14 \mathrm{E}-3$ & - & $0.14 \mathrm{E}-3$ & - & $0.14 \mathrm{E}-3$ & - \\
\hline
\end{tabular}

[3] S. Franz and T. Linß. Superconvergence analysis of the Galerkin FEM for a singularly perturbed convection-diffusion problem with characteristic layers. Numer. Methods Partial Differential Equations, 24(1):144-164, 2008.

[4] J. Li. Convergence and superconvergence analysis of finite element methods on highly nonuniform anisotropic meshes for singularly perturbed reactiondiffusion problems. Appl. Numer. Math., 36(2-3):129-154, 2001.

[5] J. Li and M. F. Wheeler. Uniform convergence and superconvergence of mixed finite element methods on anisotropically refined grids. SIAM J. Numer. Anal., 38(3):770-798, 2000.

[6] T. Linß. The necessity of Shishkin decompositions. Appl. Math. Lett., 14(7):891-896, 2001.

[7] T. Linß. Layer-adapted meshes for reaction-convection-diffusion problems, volume 1985 of Lecture Notes in Mathematics. Springer-Verlag, Berlin, 2010.

[8] R. E. O'Malley, Jr. Two-parameter singular perturbation problems for second-order equations. J. Math. Mech., 16:1143-1164, 1967. 
Table 2: $\left\|u^{I}-u^{N}\right\|_{E}$ in the case of $\varepsilon_{2}=1$

\begin{tabular}{c|ccccccccccccc}
\hline & $\varepsilon_{1}$ & $10^{0}$ & $10^{0}$ & $10^{-2}$ & $10^{-2}$ & $10^{-4}$ & $10^{-4}$ & $10^{-6}$ & $10^{-6}$ & $10^{-8}$ & $10^{-8}$ & $10^{-10}$ & $10^{-10}$ \\
& & & & & & & & & & & & \\
\hline 16 & $e_{I}^{N}$ & $p_{I}^{N}$ & $e_{I}^{N}$ & $p_{I}^{N}$ & $e_{I}^{N}$ & $p_{I}^{N}$ & $e_{I}^{N}$ & $p_{I}^{N}$ & $e_{I}^{N}$ & $p_{I}^{N}$ & $e_{I}^{N}$ & $p_{I}^{N}$ \\
32 & $0.21 \mathrm{E}-3$ & 2.00 & $0.14 \mathrm{E}-1$ & 2.04 & $0.81 \mathrm{E}-2$ & 1.84 & $0.79 \mathrm{E}-2$ & 1.82 & $0.79 \mathrm{E}-2$ & 1.82 & $0.79 \mathrm{E}-2$ & 1.82 \\
64 & $0.53 \mathrm{E}-4$ & 2.00 & $0.34 \mathrm{E}-2$ & 1.88 & $0.23 \mathrm{E}-2$ & 1.99 & $0.22 \mathrm{E}-2$ & 1.98 & $0.22 \mathrm{E}-2$ & 1.98 & $0.22 \mathrm{E}-2$ & 1.98 \\
128 & $0.13 \mathrm{E}-4$ & 2.00 & $0.91 \mathrm{E}-3$ & 1.85 & $0.57 \mathrm{E}-3$ & 2.00 & $0.57 \mathrm{E}-3$ & 2.00 & $0.57 \mathrm{E}-3$ & 2.00 & $0.57 \mathrm{E}-3$ & 2.00 \\
256 & $0.33 \mathrm{E}-5$ & 2.00 & $0.25 \mathrm{E}-3$ & 1.87 & $0.14 \mathrm{E}-3$ & 2.00 & $0.14 \mathrm{E}-3$ & 2.00 & $0.14 \mathrm{E}-3$ & 2.00 & $0.14 \mathrm{E}-3$ & 2.00 \\
512 & $0.83 \mathrm{E}-6$ & 2.00 & $0.69 \mathrm{E}-4$ & 1.92 & $0.36 \mathrm{E}-4$ & 2.00 & $0.35 \mathrm{E}-4$ & 2.00 & $0.35 \mathrm{E}-4$ & 2.00 & $0.35 \mathrm{E}-4$ & 2.00 \\
1024 & $0.21 \mathrm{E}-6$ & 2.00 & $0.18 \mathrm{E}-4$ & 1.97 & $0.89 \mathrm{E}-5$ & 2.00 & $0.89 \mathrm{E}-5$ & 2.00 & $0.89 \mathrm{E}-5$ & 2.00 & $0.89 \mathrm{E}-5$ & 2.00 \\
2048 & $0.52 \mathrm{E}-7$ & 2.00 & $0.47 \mathrm{E}-5$ & 1.99 & $0.22 \mathrm{E}-5$ & 2.00 & $0.22 \mathrm{E}-5$ & 2.00 & $0.22 \mathrm{E}-5$ & 2.00 & $0.22 \mathrm{E}-5$ & 2.00 \\
4096 & $0.13 \mathrm{E}-7$ & 2.00 & $0.12 \mathrm{E}-5$ & 2.00 & $0.56 \mathrm{E}-6$ & 2.00 & $0.55 \mathrm{E}-6$ & 2.00 & $0.55 \mathrm{E}-6$ & 2.00 & $0.55 \mathrm{E}-6$ & 2.00 \\
\hline
\end{tabular}

[9] H.-G. Roos. Error estimates for linear finite elements on Bakhvalov-type meshes. Appl. Math., 51(1):63-72, 2006.

[10] H.-G. Roos and T. Linß. Gradient recovery for singularly perturbed boundary value problems. II. Two-dimensional convection-diffusion. Math. Models Methods Appl. Sci., 11(7):1169-1179, 2001.

[11] M. Stynes and L. Tobiska. The SDFEM for a convection-diffusion problem with a boundary layer: optimal error analysis and enhancement of accuracy. SIAM J. Numer. Anal., 41(5):1620-1642, 2003.

[12] Lj. Teofanov, M. Brdar, S. Franz, and H. Zarin. SDFEM for an elliptic singularly perturbed problem with two parameters. Calcolo, 55(4):Paper No. 50, 20, 2018.

[13] Lj. Teofanov and H.-G. Roos. An elliptic singularly perturbed problem with two parameters. I. Solution decomposition. J. Comput. Appl. Math., 206(2):1082-1097, 2007.

[14] Lj. Teofanov and H. Zarin. Superconvergence analysis of a finite element method for a two-parameter singularly perturbed problem. BIT, 49(4):743$765,2009$. 
Table 3: $\left\|u-u^{N}\right\|_{E}$ in the case of $\varepsilon_{2}=10^{-4}$

\begin{tabular}{c|ccccccccccccc}
\hline$\varepsilon_{1}$ & $10^{0}$ & $10^{0}$ & $10^{-2}$ & $10^{-2}$ & $10^{-4}$ & $10^{-4}$ & $10^{-6}$ & $10^{-6}$ & $10^{-8}$ & $10^{-8}$ & $10^{-10}$ & $10^{-10}$ \\
\hline & & $e^{N}$ & $p^{N}$ & $e^{N}$ & $p^{N}$ & $e^{N}$ & $p^{N}$ & $e^{N}$ & $p^{N}$ & $e^{N}$ & $p^{N}$ & $e^{N}$ & $p^{N}$ \\
\hline 16 & $0.25 \mathrm{E}-1$ & 1.00 & $0.62 \mathrm{E}-1$ & 1.00 & $0.43 \mathrm{E}-1$ & 1.03 & $0.14 \mathrm{E}-1$ & 1.13 & $0.89 \mathrm{E}-2$ & 1.66 & $0.13 \mathrm{E}-1$ & 1.95 \\
32 & $0.13 \mathrm{E}-1$ & 1.00 & $0.31 \mathrm{E}-1$ & 1.00 & $0.21 \mathrm{E}-1$ & 1.00 & $0.66 \mathrm{E}-2$ & 1.03 & $0.28 \mathrm{E}-2$ & 1.30 & $0.33 \mathrm{E}-2$ & 1.76 \\
64 & $0.63 \mathrm{E}-2$ & 1.00 & $0.15 \mathrm{E}-1$ & 1.00 & $0.10 \mathrm{E}-1$ & 1.01 & $0.32 \mathrm{E}-2$ & 1.01 & $0.11 \mathrm{E}-2$ & 1.08 & $0.98 \mathrm{E}-3$ & 1.36 \\
128 & $0.31 \mathrm{E}-3$ & 1.00 & $0.77 \mathrm{E}-2$ & 1.00 & $0.51 \mathrm{E}-2$ & 1.01 & $0.16 \mathrm{E}-2$ & 1.00 & $0.54 \mathrm{E}-3$ & 1.02 & $0.38 \mathrm{E}-3$ & 1.07 \\
256 & $0.16 \mathrm{E}-2$ & 1.00 & $0.39 \mathrm{E}-2$ & 1.00 & $0.26 \mathrm{E}-2$ & 1.01 & $0.80 \mathrm{E}-3$ & 1.00 & $0.27 \mathrm{E}-3$ & 1.00 & $0.18 \mathrm{E}-3$ & 1.01 \\
512 & $0.78 \mathrm{E}-3$ & 1.00 & $0.19 \mathrm{E}-3$ & 1.00 & $0.13 \mathrm{E}-2$ & 1.00 & $0.40 \mathrm{E}-3$ & 1.00 & $0.13 \mathrm{E}-3$ & 1.00 & $0.90 \mathrm{E}-4$ & 1.00 \\
1024 & $0.39 \mathrm{E}-3$ & 1.00 & $0.96 \mathrm{E}-3$ & 1.00 & $0.63 \mathrm{E}-3$ & 1.00 & $0.20 \mathrm{E}-3$ & 1.00 & $0.67 \mathrm{E}-4$ & 1.00 & $0.45 \mathrm{E}-4$ & 1.00 \\
2048 & $0.20 \mathrm{E}-3$ & 1.00 & $0.48 \mathrm{E}-3$ & 1.00 & $0.32 \mathrm{E}-3$ & 1.00 & $0.10 \mathrm{E}-3$ & 1.00 & $0.33 \mathrm{E}-4$ & 1.00 & $0.23 \mathrm{E}-4$ & 1.00 \\
4096 & $0.98 \mathrm{E}-4$ & - & $0.24 \mathrm{E}-3$ & - & $0.16 \mathrm{E}-3$ & - & $0.50 \mathrm{E}-4$ & - & $0.17 \mathrm{E}-4$ & - & $0.11 \mathrm{E}-4$ & - \\
\hline
\end{tabular}

[15] J. Zhang and X. Liu. Optimal order of uniform convergence for finite element method on Bakhvalov-type meshes. J. Sci. Comput., 85(1):2, 2020.

[16] J. Zhang and X. Liu. Supercloseness of linear finite element method on Bakhvalov-type meshes for singularly perturbed convection-diffusion equation in 1D. Appl. Math. Lett., 111:106624, 7, 2021.

[17] Z. Zhang. Finite element superconvergence on Shishkin mesh for 2-D convection-diffusion problems. Math. Comp., 72(243):1147-1177, 2003. 
Table 4: $\left\|u^{I}-u^{N}\right\|_{E}$ in the case of $\varepsilon_{2}=10^{-4}$

\begin{tabular}{c|ccccccccccccc}
\hline \multirow{2}{*}{$\varepsilon_{1}$} & $10^{0}$ & $10^{0}$ & $10^{-2}$ & $10^{-2}$ & $10^{-4}$ & $10^{-4}$ & $10^{-6}$ & $10^{-6}$ & $10^{-8}$ & $10^{-8}$ & $10^{-10}$ & $10^{-10}$ \\
\hline & & & & & & & & & & & & \\
\hline 16 & $e_{I}^{N}$ & $p_{I}^{N}$ & $e_{I}^{N}$ & $p_{I}^{N}$ & $e_{I}^{N}$ & $p_{I}^{N}$ & $e_{I}^{N}$ & $p_{I}^{N}$ & $e_{I}^{N}$ & $p_{I}^{N}$ & $e_{I}^{N}$ & $p_{I}^{N}$ \\
32 & $0.37 \mathrm{E}-3$ & 2.00 & $0.11 \mathrm{E}-1$ & 1.99 & $0.24 \mathrm{E}-1$ & 1.28 & $0.12 \mathrm{E}-1$ & 2.01 & $0.14 \mathrm{E}-1$ & 2.02 & $0.17 \mathrm{E}-1$ & 2.03 \\
64 & $0.94 \mathrm{E}-4$ & 2.00 & $0.28 \mathrm{E}-2$ & 2.00 & $0.98 \mathrm{E}-2$ & 1.17 & $0.31 \mathrm{E}-2$ & 2.00 & $0.35 \mathrm{E}-2$ & 2.03 & $0.42 \mathrm{E}-2$ & 2.08 \\
128 & $0.23 \mathrm{E}-4$ & 2.00 & $0.71 \mathrm{E}-3$ & 2.00 & $0.44 \mathrm{E}-2$ & 1.30 & $0.77 \mathrm{E}-3$ & 1.92 & $0.86 \mathrm{E}-3$ & 2.02 & $0.10 \mathrm{E}-2$ & 2.14 \\
256 & $0.59 \mathrm{E}-5$ & 2.00 & $0.18 \mathrm{E}-3$ & 2.00 & $0.18 \mathrm{E}-2$ & 1.54 & $0.20 \mathrm{E}-3$ & 1.70 & $0.21 \mathrm{E}-3$ & 2.01 & $0.23 \mathrm{E}-3$ & 2.09 \\
512 & $0.15 \mathrm{E}-5$ & 2.00 & $0.45 \mathrm{E}-4$ & 2.00 & $0.61 \mathrm{E}-3$ & 1.77 & $0.63 \mathrm{E}-4$ & 1.40 & $0.52 \mathrm{E}-4$ & 2.00 & $0.54 \mathrm{E}-4$ & 2.02 \\
1024 & $0.37 \mathrm{E}-6$ & 2.00 & $0.11 \mathrm{E}-4$ & 2.00 & $0.18 \mathrm{E}-3$ & 1.91 & $0.24 \mathrm{E}-4$ & 1.31 & $0.13 \mathrm{E}-4$ & 1.99 & $0.13 \mathrm{E}-4$ & 2.00 \\
2048 & $0.92 \mathrm{E}-7$ & 2.00 & $0.28 \mathrm{E}-5$ & 2.00 & $0.47 \mathrm{E}-4$ & 1.98 & $0.97 \mathrm{E}-5$ & 1.46 & $0.33 \mathrm{E}-5$ & 1.95 & $0.33 \mathrm{E}-5$ & 1.98 \\
4096 & $0.23 \mathrm{E}-7$ & 2.00 & $0.70 \mathrm{E}-6$ & 2.00 & $0.12 \mathrm{E}-4$ & 1.99 & $0.35 \mathrm{E}-5$ & 1.68 & $0.85 \mathrm{E}-6$ & 1.84 & $0.84 \mathrm{E}-6$ & 1.94 \\
\hline
\end{tabular}

Table 5: $\left\|u-u^{N}\right\|_{E}$ in the case of $\varepsilon_{2}=10^{-8}$

\begin{tabular}{c|ccccccccccccc}
\hline$N$ & $10^{0}$ & $10^{0}$ & $10^{-2}$ & $10^{-2}$ & $10^{-4}$ & $10^{-4}$ & $10^{-6}$ & $10^{-6}$ & $10^{-8}$ & $10^{-8}$ & $10^{-10}$ & $10^{-10}$ \\
\hline & & & & & & & & & & & & \\
\hline 16 & $0.25 \mathrm{E}-1$ & 1.00 & $0.62 \mathrm{E}-1$ & 1.00 & $0.43 \mathrm{E}-1$ & 1.03 & $0.14 \mathrm{E}-1$ & 1.13 & $0.74 \mathrm{E}-2$ & 1.55 & $0.64 \mathrm{E}-2$ & 1.94 \\
32 & $0.13 \mathrm{E}-1$ & 1.00 & $0.31 \mathrm{E}-1$ & 1.00 & $0.21 \mathrm{E}-1$ & 1.01 & $0.66 \mathrm{E}-2$ & 1.03 & $0.25 \mathrm{E}-2$ & 1.23 & $0.17 \mathrm{E}-2$ & 1.74 \\
64 & $0.63 \mathrm{E}-2$ & 1.00 & $0.15 \mathrm{E}-1$ & 1.00 & $0.10 \mathrm{E}-1$ & 1.01 & $0.32 \mathrm{E}-2$ & 1.01 & $0.11 \mathrm{E}-2$ & 1.07 & $0.50 \mathrm{E}-3$ & 1.42 \\
128 & $0.31 \mathrm{E}-2$ & 1.00 & $0.77 \mathrm{E}-2$ & 1.00 & $0.51 \mathrm{E}-2$ & 1.01 & $0.16 \mathrm{E}-2$ & 1.00 & $0.51 \mathrm{E}-3$ & 1.02 & $0.19 \mathrm{E}-3$ & 1.16 \\
256 & $0.16 \mathrm{E}-2$ & 1.00 & $0.39 \mathrm{E}-2$ & 1.00 & $0.26 \mathrm{E}-2$ & 1.01 & $0.80 \mathrm{E}-3$ & 1.00 & $0.25 \mathrm{E}-3$ & 1.01 & $0.83 \mathrm{E}-4$ & 1.05 \\
512 & $0.78 \mathrm{E}-3$ & 1.00 & $0.19 \mathrm{E}-3$ & 1.00 & $0.13 \mathrm{E}-2$ & 1.00 & $0.40 \mathrm{E}-3$ & 1.00 & $0.13 \mathrm{E}-3$ & 1.00 & $0.40 \mathrm{E}-4$ & 1.01 \\
1024 & $0.39 \mathrm{E}-3$ & 1.00 & $0.96 \mathrm{E}-3$ & 1.00 & $0.63 \mathrm{E}-3$ & 1.00 & $0.20 \mathrm{E}-3$ & 1.00 & $0.63 \mathrm{E}-4$ & 1.00 & $0.20 \mathrm{E}-4$ & 1.00 \\
2048 & $0.20 \mathrm{E}-3$ & 1.00 & $0.48 \mathrm{E}-3$ & 1.00 & $0.32 \mathrm{E}-3$ & 1.00 & $0.10 \mathrm{E}-3$ & 1.00 & $0.32 \mathrm{E}-4$ & 1.00 & $0.10 \mathrm{E}-4$ & 1.00 \\
4096 & $0.98 \mathrm{E}-4$ & - & $0.24 \mathrm{E}-3$ & - & $0.16 \mathrm{E}-3$ & - & $0.50 \mathrm{E}-4$ & - & $0.16 \mathrm{E}-4$ & - & $0.50 \mathrm{E}-5$ & - \\
\hline
\end{tabular}


Table 6: $\left\|u^{I}-u^{N}\right\|_{E}$ in the case of $\varepsilon_{2}=10^{-8}$

\begin{tabular}{|c|c|c|c|c|c|c|c|c|c|c|c|c|}
\hline & $10^{0}$ & $10^{0}$ & $10^{-2}$ & $10^{-2}$ & $10^{-4}$ & $10^{-4}$ & $10^{-6}$ & $10^{-6}$ & $10^{-8}$ & $10^{-8}$ & $10^{-10}$ & $10^{-10}$ \\
\hline & $e_{I}^{N}$ & $p_{I}^{N}$ & $e_{I}^{N}$ & $p_{I}^{N}$ & $e_{I}^{N}$ & $p_{I}^{N}$ & $e_{I}^{N}$ & $p_{I}^{N}$ & $e_{I}^{N}$ & $p_{I}^{N}$ & $e_{I}^{N}$ & $p_{I}^{N}$ \\
\hline 16 & $0.37 \mathrm{E}-3$ & 1.99 & $0.11 \mathrm{E}-1$ & 1.99 & $0.24 \mathrm{E}-1$ & 1.28 & $0.12 \mathrm{E}-1$ & 2.01 & $0.13 \mathrm{E}-1$ & 2.00 & $0.14 \mathrm{E}-1$ & 2.00 \\
\hline 32 & $0.94 \mathrm{E}-4$ & 2.00 & $0.28 \mathrm{E}-2$ & 2.00 & $0.98 \mathrm{E}-2$ & 1.17 & $0.31 \mathrm{E}-2$ & 2.00 & $0.34 \mathrm{E}-2$ & 2.00 & $0.34 \mathrm{E}-2$ & 2.00 \\
\hline 64 & $0.23 \mathrm{E}-4$ & 2.00 & $0.71 \mathrm{E}-3$ & 2.00 & $0.44 \mathrm{E}-2$ & 1.30 & $0.77 \mathrm{E}-3$ & 1.92 & $0.84 \mathrm{E}-3$ & 1.98 & $0.85 \mathrm{E}-3$ & 2.00 \\
\hline 128 & $0.59 \mathrm{E}-5$ & 2.00 & $0.18 \mathrm{E}-3$ & 2.00 & $0.18 \mathrm{E}-2$ & 1.54 & $0.20 \mathrm{E}-3$ & 1.70 & $0.21 \mathrm{E}-3$ & 2.01 & $0.21 \mathrm{E}-3$ & 1.99 \\
\hline 256 & $0.15 \mathrm{E}-5$ & 2.00 & $0.45 \mathrm{E}-4$ & 2.00 & $0.61 \mathrm{E}-3$ & 1.77 & $0.63 \mathrm{E}-4$ & 1.40 & $0.53 \mathrm{E}-4$ & 2.01 & $0.53 \mathrm{E}-4$ & 1.98 \\
\hline 512 & $0.37 \mathrm{E}-6$ & 2.00 & $0.11 \mathrm{E}-4$ & 2.00 & $0.18 \mathrm{E}-3$ & 1.91 & $0.24 \mathrm{E}-4$ & 1.31 & $0.13 \mathrm{E}-4$ & 2.00 & $0.14 \mathrm{E}-4$ & 2.02 \\
\hline 1024 & $0.92 \mathrm{E}-7$ & 2.00 & $0.28 \mathrm{E}-5$ & 2.00 & $0.47 \mathrm{E}-4$ & 1.98 & $0.96 \mathrm{E}-5$ & 1.45 & $0.33 \mathrm{E}-5$ & 1.98 & $0.33 \mathrm{E}-5$ & 2.01 \\
\hline 2048 & $0.23 \mathrm{E}-7$ & 2.00 & $0.70 \mathrm{E}-6$ & 2.00 & $0.12 \mathrm{E}-4$ & 1.99 & $0.35 \mathrm{E}-5$ & 1.68 & $0.83 \mathrm{E}-6$ & 1.92 & $0.83 \mathrm{E}-6$ & 2.00 \\
\hline 4096 & $0.57 \mathrm{E}-8$ & - & $0.17 \mathrm{E}-6$ & - & $0.30 \mathrm{E}-5$ & - & $0.11 \mathrm{E}-5$ & - & $0.22 \mathrm{E}-6$ & - & $0.21 \mathrm{E}-6$ & - \\
\hline
\end{tabular}

